\title{
TAUBERIAN THEOREMS FOR LOGARITHMIC SUMMABILITY (L)
}

\author{
M. S. RANGACHARI AND Y. SitARAMAN
}

(Received March 23, 1964)

1. Introduction. For a series $\sum a_{n}$ or sequence $s_{n}(n=0,1,2, \cdots)$ of its partial sums, there is a generalization of Abel or $(A)$ summability which has been defined as follows: if

For any $\alpha>-1,\left\{s_{n}\right\}$ is summable $\left(A_{\alpha}\right)$ to $s$ (finite), or briefly, $s_{n} \rightarrow s\left(A_{\alpha}\right)$,

$$
(1-x)^{\alpha+1} \sum_{n=0}^{\infty}\left(\begin{array}{c}
n+\alpha \\
n
\end{array}\right) s_{n} x^{n} \rightarrow s \quad \text { as } x \rightarrow 1-0,
$$

the series in (1) being supposed to converge for $0<x<1$. The particular case of $\left(A_{\alpha}\right)$ summability with $\alpha=0$ is $(A)$ summability and the limiting case with $\alpha=-1$, separately defined below after Borwein [1], may be called $(L)$ summability after Ishiguro ([3], [4]).

$\left\{s_{n}\right\}$ is summable $(L)$ to $s$, or $s_{n} \rightarrow s(L)$, if

$$
-\frac{1}{\log (1-x)} \sum_{n=0}^{\infty} \frac{s_{n}}{n+1} x^{n+1} \rightarrow s \quad \text { as } x \rightarrow 1-0,
$$

the convergence of the series in (2) being assumed for $0<x<1$. Summability $\left(A_{\alpha}\right)$ introduced independently by Jakimovski ([5], p. 374) and Rajagopal ([7], p. 93), has been considered in detail by Borwein [1], while summability $(L)$ mentioned by Hardy $([2]$, p. 81$)$ in one connexion and by Rajagopal ([7], p. 94) in another, has been subsequently studied, first by Borwein [1] in respect of its relation to summability $\left(A_{\alpha}\right), \alpha>-1$, as well as certain other summabilities, and then by Ishiguro independently (loc. cit.) from the point of view of Tauberian theory. It is the object of this paper to continue Ishiguro's work by presenting three Tauberian theorems for summability $(L)$, numbered $\mathrm{I}(L), \mathrm{II}(L)$ and $\mathrm{III}(L)$ in the sequel, analogous to well-known theorems for Abel summability and their extensions to summability $\left(A_{\alpha}\right)$.

In a discussion of the Tauberian theorems last mentioned, the methods of summability $\left(A_{\alpha}\right)$ for all $\alpha>-1$ can in general be uniformly treated, so that the treatment includes as a special case the Abel method $\left(A_{o}\right)$ or $(A)$. However, such a uniform treatment of the $\left(A_{\alpha}\right)$ methods cannot always be 
extended to the $(L)$ method. Either a known basic proposition to which we appeal in the case of the $\left(A_{\alpha}\right)$ methods has to be modified in details if not in essentials to make it applicable to the $(L)$ method (e.g. Proposition 1 or 2 infra), or else the basic proposition for the $\left(A_{\alpha}\right)$ methods is not applicable at all to the $(L)$ method. The first alternative is illustrated by the proofs of Theorems I $(L)$, III $(L)$ and the second alternative by the proof of Theorem II $(L)^{*}$. It should be mentioned at the same time that the proofs of these particular theorems for $(L)$ summability suggest similar proofs of the analogous Theorems I $(A), \operatorname{II}(A)$, III $(A)$ for $\left(A_{\alpha}\right)$ summability, though they may not be useful pointers to proofs of other theorems for $\left(A_{\alpha}\right)$ summability or to proofs of analogous theorems for other summabilities.

The authors would like to acknowledge here, their indebtedness to Prof. C. T. Rajagopal for help in the preparation of their paper and to Dr. B. Kuttner for a kindly scrutiny of their original manuscripts which has resulted in a correction.

2. Preliminary results. The methods of summability $\left(\mathrm{A}_{\alpha}\right), \alpha>-1$, and summability $(L)$ are particular cases of a positive regular method of summability $(\tau)$ defined thus:

$$
\left\{\begin{array}{l}
\text { If, for } n=0,1,2, \cdots, c_{n}(y) \geqq 0(y>0), c_{n}(y) \rightarrow 0(y \rightarrow \infty), \sum_{n=0}^{\infty} c_{n}(y)=1, \\
\left\{s_{n}\right\} \text { is such that } \tau(y)=\sum_{n=0}^{\infty} c_{n}(y) s_{n} \text { converges for } y>0, \\
\text { then }\left\{s_{n}\right\} \text { is summable }(\tau) \text { to } s \text { when } \tau(y) \rightarrow s \text { as } y \rightarrow \infty .
\end{array}\right.
$$

Summability $(\tau)$ as defined by (3) reduces to summability $\left(A_{\alpha}\right)$ as defined by (1) or summability $(L)$ as defined by (2), according as

or

$$
\left.\begin{array}{l}
c_{n}(y)=\alpha_{n}(y) \equiv\left(1-e^{-1 / y}\right)^{\alpha+1}\left(\begin{array}{c}
n+\alpha \\
n
\end{array}\right) e^{-n / y} \\
c_{n}(y)=\lambda_{n}(y) \equiv \frac{-e^{-(n+1) / y}}{\log \left(1-e^{-1 / y}\right)(n+1)}
\end{array}\right\} e^{-1 / y}=x
$$

If the transform $\tau(y)$ of (3) corresponding to the $c_{n}$ 's of (4) and (5) be denoted respectively by $A_{\alpha}(y)$ and $L(y)$ the definitions in (1) and (2) can be restated thus :

* Theorem $\mathrm{I}(L)$ is the first author's and Theorem $\mathrm{II}(L)$, III $(L)$, are the second author's. The theorems, with their authorship thus specified, formed the substance of two communications to the 29th Conference of the Indian Mathematical Society held at Madras in December 1963. As for Theorems $\mathrm{I}(A), \operatorname{II}(A), \operatorname{III}(A)$, full details of their proofs are supplied in an unpublished paper of the second author's. 


$$
\begin{array}{lll}
s_{n} \rightarrow s\left(A_{\alpha}\right) \quad \text { if } & A_{\alpha}(y) \equiv \sum_{n=0}^{\infty} \alpha_{n}(y) s_{n} \rightarrow s & \text { as } y \rightarrow \infty, \\
s_{n} \rightarrow s(L) \quad \text { if } & L(y) \equiv \sum_{n=0}^{\infty} \lambda_{n}(y) s_{n} \rightarrow s & \text { as } y \rightarrow \infty,
\end{array}
$$

where $\alpha_{n}(y)$ and $\lambda_{n}(y)$ have the definitions in (4) and (5).

The following two propositions for the transform $\tau(y)$ are modifications of known results, required for our purpose.

Proposition 1. Suppose that

(i ) $\phi(u)$ is a positive monotonic increasing unbounded function of $u \geqq 1$ such that

$$
\Phi(u) \equiv \int_{u_{0}}^{u} \frac{d t}{\phi(t)} \rightarrow \infty \quad \text { if } \quad 1 \leqq u_{0}<u \rightarrow \infty
$$

(ii) the functions $c_{n}(y)$ satisfy the following conditions in addition to the conditions at the beginning of (3):

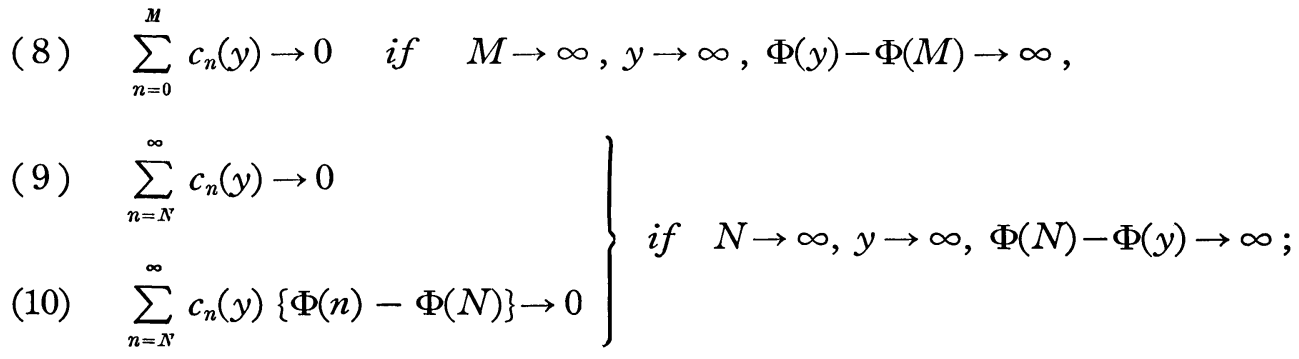

(iii) $\left\{s_{n}\right\}$ is such that there are positive numbers $a$ and $b$ which make

$$
s_{q}-s_{p}>-a\{\Phi(q)-\Phi(p)\}-b \text { for } q>p>p_{0} .
$$

Then $\tau(y)=O(1)(y \rightarrow \infty)$ implies $s_{n}=O(1)(n \rightarrow \infty)$.

Proposition 1 is a modified form of a principle due to Vijayaraghavan, as stated by Hardy ([2], p. 306, Theorem 238), and is proved exactly like that principle.

Proposition 2. Suppose that, in Proposition 1, (ii) is replaced by a condition as follows:

$y$ and positive integers $M, N$ are related so that

$$
\Phi(y)-\Phi(M)=\mu>0, \Phi(N)-\Phi(y)=\nu>0,
$$

and, corresponding to any small $\epsilon>0$, we have: 


$$
\sum_{n=0}^{M} c_{n}(y)<\epsilon
$$

(9') $\quad \sum_{n=N}^{\infty} c_{n}(y)<\epsilon$

for all large enough $y, M, N, \mu, \nu$,

$$
\sum_{n=N}^{\infty} c_{n}(y)\{\Phi(n)-\Phi(N)\}<\epsilon
$$

and additionally, for any choice of $\mu, \nu$ suitably large and all large enough $y, M, N$

$$
\sum_{n=M}^{\infty} c_{n}(y)|\Phi(n)-\Phi(p)|<K(\mu, \nu), \text { where } p=M \text { or } N
$$

Suppose also that hypothesis (iii) of Proposition 1 is changed to

$$
s_{p}-s_{q}=o_{L}(1)\{\Phi(q)-\Phi(p)\}+o_{L}(1), \quad q>p \rightarrow \infty .
$$

Then the conclusion of Proposition 1 can be refined as follows:

$$
\underset{y \rightarrow \infty}{\operatorname{Osc} \tau} \tau(y)=\underset{n \rightarrow \infty}{\operatorname{Osc}} s_{n}<\infty,
$$

i.e.

$$
\lim _{y \rightarrow \infty} \sup _{\text {inf }} \tau(y)=\lim _{n \rightarrow \infty} \sup _{\text {inf }} s_{n},
$$

all the limits being finite.

Proposition 2 is a companion to Proposition 1, proved just like its original version, in a somewhat different form, given by Rajagopal ([8], Theorem B).

PROPOSITION 3. If $\left\{s_{n}\right\}$ is real, $s_{n} \rightarrow s(L)$ in the sense of (7) and $s_{n}$ is bounded on one side, then

$$
\left\{\begin{aligned}
t_{m} & =\left(s_{o}+\frac{s_{1}}{2}+\cdots+\frac{s_{m}}{m+1}\right) /\left(1+\frac{1}{2}+\cdots+\frac{1}{m+1}\right) \\
& \sim \frac{1}{\log m}\left(s_{o}+\frac{s_{1}}{2}+\cdots+\frac{s_{m}}{m+1}\right) \rightarrow s(m \rightarrow \infty)
\end{aligned}\right.
$$

which relation may be said to define summability $(l)$ of $\left\{s_{n}\right\}$ to $s$ and written $s_{n} \rightarrow s(l)$.

This proposition has been proved by Ishiguro ([3], Theorem 2).

PROPOSITION 4. (i) Summability (l) of $\left\{s_{n}\right\}$ to $s$ in the sense of (14) is 
equivalent to summability $(R, \log (n+1), 1)$ of $\left\{s_{n}\right\}$ to $s$ in the usual sense ([2], p. 86) :

(ii) Summability (l) of $\left\{s_{n}\right\}$ to $s$ and

$$
\liminf _{n \rightarrow \infty}\left(s_{n}-s_{n-1}\right) n \log n=-w \quad(0 \leqq w<\infty)
$$

together imply the convergence of $\left\{s_{n}\right\}$ to $s$.

Part (i) of Proposition 4 is well known ([2], p. 87, Theorem 37) and part (ii) follows from (i) by a familiar Tauberian theorem ([2], Theorem 67 and p. 145 , Notes).

3. Theorems. In all the theorems which follow, $\left\{s_{n}\right\}$ is a real sequence. Of the immediately following pair of analogous theorems for summabilities $\left(A_{\alpha}\right)$ and $(L)$, the second is proved and the first is merely stated as it can be proved similarly. In the case $\alpha=0$ the first theorem is classical ([2], p. 154, Theorem 91).

THEOREM I $(A)$. If $\left\{s_{n}\right\}$ is summable $\left(A_{\alpha}\right)$ to $s$ and such that

$$
\liminf _{n \rightarrow \infty}\left(s_{n}-s_{n-1}\right) n=-w \quad(0 \leqq w<\infty),
$$

then $s_{n}$ is convergent to $s$.

THEOREM I $(L)$. If $\left\{s_{n}\right\}$ is summable $(L)$ to $s$ and such that

$$
\liminf _{n \rightarrow \infty}\left(s_{n}-s_{n-1}\right) n \log n=-w \quad(0 \leqq w<\infty),
$$

then $s_{n}$ is convergent to $s$.

Ishiguro ([4], Theorem 2) has proved Theorem $\mathrm{I}(L)$ in the special case $\lim \left(s_{n}-s_{n-1}\right) n \log n=0$, of (16). Theorem I $(L)$ as stated follows obviously from the lemma given below taken along with Proposition 3 and Proposition 4 successively.

LEMMA (L). If $L(y)$ is defined as in (7) and bounded as $y \rightarrow \infty$ or in particular, convergent to $s$ as $y \rightarrow \infty$, then hypothesis (16) ensures that $s_{n}$ is bounded.

This lemma may be deduced thus from Proposition 1 . We first observe that the hypothesis (16) of the lemma ensures hypothesis (11) of Proposition 1 with $\phi(u)=u \log u$. The remaining hypotheses (8), (9), (10) of Proposition 1 are also ensured by the $c_{n}(y)=\lambda_{n}(y)$ of (5) and the $\Phi(u)$ corresponding to 
our choice of $\phi(u)$, viz.

$$
\Phi(u)=\int_{e}^{u} \frac{d t}{\phi(t)}=\int_{e}^{u} \frac{d t}{t \log t}=\log \log u
$$

when $y, M, N$ are such that, as $y \rightarrow \infty$,

$$
\left\{\begin{array}{cl}
\Phi(y)-\Phi(M)=\log \frac{\log y}{\log M} \rightarrow \infty & \text { or } \quad \frac{\log y}{\log M} \rightarrow \infty \\
\Phi(N)-\Phi(y)=\log \frac{\log N}{\log y} \rightarrow \infty & \text { or } \quad \frac{\log y}{\log N} \rightarrow 0
\end{array}\right.
$$

In the first place, we get from (5) and (17), as $y \rightarrow \infty$,

$$
\begin{aligned}
\sum_{0}^{M} \lambda_{n}(y) & =-\frac{1}{\log \left(1-e^{-1 / y}\right)} \sum_{0}^{M} \frac{e^{-(n+1) / y}}{n+1} \\
& \sim \frac{1}{\log y} \sum_{0}^{M} \frac{e^{-(n+1) / y}}{n+1}<\frac{1}{\log y} \sum_{0}^{M} \frac{1}{n+1} \sim \frac{\log M}{\log y}=o(1) .
\end{aligned}
$$

Next, since we may suppose that $y<N$ as $y \rightarrow \infty$,

$$
\sum_{N^{\prime}}^{\infty} \lambda_{n}(y) \sim \frac{1}{\log y} \sum_{N^{N}}^{\infty} \frac{e^{-(n+1) / y}}{n+1}<\frac{1}{\log y} \cdot \frac{1}{N} \cdot \frac{e^{-\left(N^{N}+1\right) / y}}{1-e^{-1 / y}} \sim \frac{1}{\log y} \cdot \frac{y}{N}=o(1) .
$$

Finally, using the fact that $\log x \leqq x-1(x>0)$, we get when $y \rightarrow \infty$,

$$
\begin{aligned}
\sum_{N}^{\infty} \lambda_{n}(y)\{\Phi(n)-\Phi(N)\} & \sim \frac{1}{\log y} \sum_{N}^{\infty} \frac{e^{-(n+1) / y}}{n+1} \log \frac{\log n}{\log N} \\
& <\frac{1}{\log y} \sum_{N}^{\infty} \frac{e^{-(n+1) / y}}{n+1}\left(\frac{\log n}{\log N}-1\right) \\
& =\frac{1}{\log y \cdot \log N} \sum_{N}^{\infty} \frac{e^{-(n+1) / y}}{n+1} \log (n / N) \\
& <\frac{1}{\log y \cdot \log N} \sum_{\nu=0}^{\infty} \frac{e^{-(N+\nu) / y}}{N+\nu} \log \left(\frac{\nu}{N}+1\right) \\
& <\frac{1}{\log y \cdot \log N} \sum_{0}^{\infty} \frac{e^{-(N+\nu) / y}}{N+\nu} \cdot \frac{\nu}{N} \\
& <\frac{e^{-N / y}}{\log y \cdot \log N \cdot N^{2}} \sum_{0}^{\infty} \nu e^{-\nu / y}
\end{aligned}
$$




$$
\begin{aligned}
& =\frac{e^{-N / y}}{\log y \cdot \log N \cdot N^{2}}\left(1-e^{-1 / y}\right)^{-2} \\
& \sim \frac{e^{-N / y}}{\log y \cdot \log N}\left(\frac{y}{N}\right)^{2}<\frac{1}{\log y \cdot \log N}=o(1) .
\end{aligned}
$$

Thus the hypotheses of the lemma imply the hypotheses of Proposition 1 in a special case and the conclusion of boundedness of $s_{n}$ in the proposition can be carried over to the lemma.

A second pair of analogous theorems for summabilties $\left(A_{\alpha}\right)$ and $(L)$ is given below. Alternative (i) of each of these theorems can be treated as a special case of Proposition 1. In fact, alternative (i) of Theorem II $(L)$ has been so treated in Lemma $(L)$, and alternative (i) of Theorem $\operatorname{II}(A)$ can be similarly treated.

THEOREM II $(A)$. Suppose that $\left\{s_{n}\right\}$ satisfies the condition (15) and that the transform $A_{\alpha}(y)$ of $\left\{s_{n}\right\}$ defined in (6) satisfies the condition

$$
\limsup _{y \rightarrow \infty} A_{\alpha}(y)<\infty \text {. }
$$

Then

$$
\begin{gathered}
\text { either (i ) } \quad \begin{array}{cl}
\liminf _{y \rightarrow \infty} A_{\alpha}(y)>-\infty, & \text { with } s_{n}=O(1), \\
\text { or (ii) } \quad & \liminf _{y \rightarrow \infty} A_{\alpha}(y)=-\infty, \quad \text { with } \\
s_{n} \leqq[w+o(1)] \log \log n \quad(n \rightarrow \infty)
\end{array}
\end{gathered}
$$

THEOREM II $(L)$. Suppose that $\left\{s_{n}\right\}$ is subject to the condition (16) and that the transform $L(y)$ of $\left\{s_{n}\right\}$ defined in (7) is subject to the condition

$$
\limsup _{y \rightarrow \infty} L(y)<\infty \text {. }
$$

Then

$$
\begin{aligned}
& \text { either ( i ) } \quad \liminf _{y \rightarrow \infty} L(y)>-\infty \text {, with } s_{n}=O(1) \text {, } \\
& \text { or (ii) } \quad \liminf _{y \rightarrow \infty} L(y)=-\infty, \text { with } \\
& s_{n} \leqq[w+o(1)] \log \log \log n \quad(n \rightarrow \infty) .
\end{aligned}
$$

In the case of alternative (ii) of each of the above theorems, for which a proof has not been indicated, the following points may be noted. Alternative (ii) of Theorem II $(A)$, in the case $\alpha=0$, is due to Karamata [6] who also showed that (19) then gives the best possible upper estimate for $s_{n}$ in that 
$\log \log n$ on the right side of (19) is not always replaceable by a smaller function of $n$. Further, alternative (ii) of Theorem $\mathrm{II}(A)$ for all $\alpha>-1$ is deducible from a general result which is a complement to Proposition 1, obtained by Rajagopal and Vijayaraghavan ([8], Corollary 1 under Theorem 1). But alternative (ii) of Theorem II $(L)$ is not so deducible and is proved below $a b$ initio.

We first note that, by (16) we can find a positive integer $M_{o}$, corresponding to any small $\epsilon>0$ such that

$$
\begin{aligned}
s_{n}-s_{M}=\sum_{r=M+1}^{n}\left(s_{r}-s_{r-1}\right) & >-(w+\epsilon) \sum_{M+1}^{n} \frac{1}{r \log r} \quad\left(n>M>M_{o}\right) \\
& >-(w+\epsilon) \int_{M}^{n} \frac{d u}{u \log u} \\
& =-(w+\epsilon)(\log \log n-\log \log M) .
\end{aligned}
$$

We next observe that, by (16) again, there is a constant $K>0$ such that

$$
\begin{aligned}
s_{n}=s_{2}+\sum_{r=3}^{n}\left(s_{r}-s_{r-1}\right) & >s_{2}-K \sum_{r=3}^{n} \frac{1}{r \log r} \quad(2 \leqq n<M) \\
& >s_{2}-K \int_{2}^{n} \frac{d u}{u \log u} \\
& =K^{\prime}-K \log \log n>K^{\prime}-K \log \log M,
\end{aligned}
$$

where $K^{\prime}=s_{2}+K \log \log 2$. Recalling the defintion of $L(y)$ in (7) we can write :

$$
s_{M} \sum_{n=M}^{\infty} \lambda_{n}(y)=L(y)-\sum_{n=0}^{M-1} s_{n} \lambda_{n}(y)-\sum_{n=M}^{\infty}\left(s_{n}-s_{M}\right) \lambda_{n}(y),
$$

and then use (23) in the second term on the right side and (22) in the third term. The result is

$$
\begin{aligned}
s_{M} \sum_{n=M}^{\infty} \lambda_{n}(y)<L(y)-K^{\prime} \sum_{0}^{M-1} \lambda_{n}(y) & +K \log \log M \sum_{o}^{M-1} \lambda_{n}(y)+ \\
& +(w+\epsilon) \sum_{M}^{\infty}(\log \log n-\log \log M) \lambda_{n}(y) .
\end{aligned}
$$

On the right side, the first term is by hypothesis (20) bounded above and the factor multiplying $K^{\prime}$ in the second term is positive and less than unity from the defintion of $\lambda_{n}(y)$ in (5). Hence these two terms are together less 
than a positive constant $\mathrm{K}^{\prime \prime}$, and we get

$$
\begin{gathered}
s_{M}<\frac{K^{\prime \prime}}{1-\sum_{0}^{M-1} \lambda_{n}(y)}+K \log \log M \sum_{0}^{M-1} \frac{\lambda_{n}(y)}{1-\sum_{0}^{M-1} \lambda_{n}(y)}+ \\
+\frac{w+\epsilon}{1-\sum_{0}^{M-1} \lambda_{n}(y)} \sum_{M}^{\infty}(\log \log n-\log \log M) \lambda_{n}(y) \\
\text { (24) }=\sum_{1}+\sum_{2}+\sum_{3} \quad \text { (say). }
\end{gathered}
$$

In each of the three terms on the right side of (24),

$$
\begin{aligned}
\sum_{o}^{M-1} \lambda_{n}(y) & =-\frac{1}{\log \left(1-e^{-1 / y}\right)} \sum_{o}^{M-1} \frac{e^{-(n+1) / y}}{n+1} \\
& <\frac{1}{\log y} \sum_{0}^{M-1} \frac{1}{n+1}<\frac{\log M+C}{\log y}<1
\end{aligned}
$$

where $C$ is a positive constant, if

$$
\log y=\log M \log \log M \quad\left(M>M_{1}\right)
$$

which is a choice of $y$ (for all suitable large $M$, as stated) adopted throughout in the rest of this proof. Using (25) and (26) in the terms $\sum_{1}$ and $\sum_{2}$ of (24), we get

(27) $\sum_{1}=\frac{K^{\prime \prime}}{1-\sum_{0}^{M-1} \lambda_{n}(y)}<\frac{K^{\prime \prime} \log y}{\log y-\log M-C} \sim K^{\prime \prime} \quad(M \rightarrow \infty)$,

(28) $\sum_{2}=K \log \log M \frac{\sum_{0}^{M-1} \lambda_{n}(y)}{1-\sum_{0}^{M-1} \lambda_{n}(y)}<K \log \log M \frac{\log M+C}{\log y-\log M-C} \sim K$

Also,

$$
\begin{aligned}
\sum_{3} & =\frac{w+\epsilon}{1-\sum_{0}^{M-1} \lambda_{n}(y)} \cdot \frac{-1}{\log \left(1-e^{-1 / y}\right)} \sum_{M}^{\infty} \frac{\log \log n-\log \log M}{n+1} e^{-(n+1) / y} \\
& <\frac{w+\epsilon}{1-\sum_{0}^{M-1} \lambda_{n}(y)} \cdot \frac{1}{\log y} \int_{M}^{\infty} \frac{\log \log u-\log \log M}{u} e^{-u / y} d u \quad\left(M>M_{0}\right) .
\end{aligned}
$$


Changing the variable in the last integral to $v=u / y$, we get

$$
\begin{aligned}
\sum_{3} & <\frac{w+\epsilon}{1-\sum_{0}^{M-1} \lambda_{n}(y)} \cdot \frac{1}{\log y} \int_{M / y}^{\infty} \frac{\log \log y v-\log \log M}{v} e^{-v} d v \\
& <\frac{w+\epsilon}{1-\sum_{o}^{M-1} \lambda_{n}(y)} \cdot \frac{1}{\log y}\left\{(\log \log y-\log \log M) \int_{M / y}^{1} \frac{d v}{v}+\right. \\
& \left.\quad+\int_{1}^{y} \frac{\log \log y^{2}-\log \log M}{v} e^{-v} d v+\int_{y}^{\infty} \frac{\log \log v^{2}}{v} e^{-v} d v\right\} \\
\text { (30) }= & +\sum_{31}+\sum_{33} \cdot
\end{aligned}
$$

Using (25) and (26) in $\sum_{31}, \sum_{32}$ and $\sum_{33}$, we find that

$$
\begin{aligned}
\sum_{31} & <\frac{(w+\epsilon) \log y}{\log y-\log M-C} \cdot \frac{\log \log y-\log \log M}{\log y}(\log y-\log M) \\
& \sim(w+\epsilon) \log \log \log M \quad(M \rightarrow \infty) \\
\sum_{32} & <(w+\epsilon) \frac{\log y}{\log y-\log M-C} \cdot \frac{\log 2+\log \log y-\log \log M}{\log y} \int_{1}^{\infty} \frac{e^{-v}}{v} d v
\end{aligned}
$$$$
\text { (32) }=o(1) \quad(M \rightarrow \infty) \text {, }
$$

(33) $\sum_{33}<(w+\epsilon) \frac{\log y}{\log y-\log M-C} \cdot \frac{1}{\log y} \int_{y}^{\infty} \frac{\log \log v^{2}}{v} e^{-v} d v=o(1)$

$$
(M \rightarrow \infty) \text {. }
$$

Using (31), (32), (33) in (30), we get $\sum_{3}<(w+2 \epsilon) \log \log \log M$ for all large $M$. Then using the last relation together with (27) and (28) in (24) we reach the desired conclusion (21) with $M$ in place of $n$ :

$$
s_{M} \leqq[w+o(1)] \log \log \log M \quad(M \rightarrow \infty) .
$$

REMARK ON THEOREM II $(L)$. The omission of the Tauberian condition (16) may imply an upper estimate for $s_{M}$ larger than the one reached above, as shown by the example

$$
s_{n}=(-1)^{n}(n+1)^{1 / 2}-\left[1-(-1)^{n}\right](n+1) .
$$

The last pair of analogues in this paper, for summabilities $\left(A_{\alpha}\right)$ and $(L)$, are the Tauberian theorems which follows. They are refinements of the 
alternatives (i) of Theorems II $(A)$ and II $(L)$, deducible from Proposition 2 by similar arguments. For the reason last stated, the deduction of the theorem for $(L)$ summability and that for $\left(A_{\alpha}\right)$ summability is omitted.

THEOREM III $(A)$. If $\left\{s_{n}\right\}$ satisfies the condition

$$
\liminf _{n \rightarrow \infty}\left(s_{n}-s_{n-1}\right) n=0,
$$

and the transform $A_{\alpha}(y)$ of $\left\{s_{n}\right\}$ in (6) is bounded as $y \rightarrow \infty$, then

$$
\underset{n \rightarrow \infty}{\operatorname{Osc}} s_{n}=\underset{y \rightarrow \infty}{\operatorname{Osc}} A_{\alpha}(y) \text {. }
$$

THEOREM III $(L)$. If $\left\{s_{n}\right\}$ is subject to the condition

$$
\liminf _{n \rightarrow \infty}\left(s_{n}-s_{n-1}\right) n \log n=0,
$$

and the transform $L(y)$ of $\left\{s_{n}\right\}$ in (7) is bounded as $y \rightarrow \infty$, then

$$
\underset{n \rightarrow \infty}{\operatorname{Osc}} s_{n}=\underset{y \rightarrow \infty}{\operatorname{Osc}} L(y) \text {. }
$$

We deduce Theorem III $(L)$ from Proposition 2 on the lines of our deduction of Lemma $(L)$ from Proposition 1 . We first note that hypothesis (35) implies hypothesis (13) of Proposition 2 with $\phi(u)=u \log u$. As in the derivation of Lemma $(L)$ from Proposition 1 , the $c_{n}(y)=\lambda_{n}(y)$ of $(5)$ and the $\Phi(u)=\log \log u$ corresponding to our choice of $\phi(u)$ can be shown to satisfy hypotheses (8), (9') and (10') of Proposition 2. Theorem III $(L)$ will follow as a special case of Proposition 2 if we show further that our choices of $c_{n}(y)$ and $\phi(u)$ or $\Phi(u)$ satisfy hypothesis (12) of Proposition 2 under the accompanying condition that $y, M, N$ may tend to $\infty$ but are such that

$$
\left\{\begin{array}{l}
\Phi(y)-\Phi(M)=\log \frac{\log y}{\log M}=\mu>\mu_{0} \\
\Phi(N)-\Phi(y)=\log \frac{\log N}{\log y}=\nu>\nu_{0}
\end{array} \quad(\mu, \nu \text { fixed }) .\right.
$$

We begin by considering

$$
\begin{aligned}
\sum_{n=M}^{\infty} c_{n}(y)|\Phi(n)-\Phi(N)| & =\sum_{n=M}^{N} \lambda_{n}(y)\{\Phi(N)-\Phi(n)\}+\sum_{n=N+1}^{\infty} \lambda_{n}(y)\{\Phi(n)-\Phi(N)\} \\
& =S_{1}+S_{2} \text { (say). }
\end{aligned}
$$

$S_{2}$ has already been considered in the proof of Lemma $(L)$ and shown to be arbitrarily small for all sufficiently large $y, M, N, \mu, \nu$. Also, 


$$
S_{1}=\sum_{n=M}^{N} \lambda_{n}(y)\{\Phi(N)-\Phi(n)\} \sim \frac{1}{\log y} \sum_{n=M}^{N} \frac{\log \log N-\log \log n}{n+1} e^{-(n+1) / y} \quad(y \rightarrow \infty) .
$$

An obvious upper estimate for the extreme right-hand member, under the condition (36), is given by

$$
\begin{aligned}
\frac{\log \log N-\log \log M}{\log y} \sum_{n=M}^{N} \frac{1}{n+1} & =\log \frac{\log N}{\log M} \cdot \frac{\log N-\log M+o(1)}{\log y} \\
& =(\mu+\nu)\left[e^{\nu}-e^{-\mu}+o(1)\right] .
\end{aligned}
$$

Hence, using in (37) the upper estimates for $S_{1}$ and $S_{2}$ obtained above, we get, with our choice of $c_{n}(y)$ and $\Phi(u)$,

$$
\sum_{n=M}^{\infty} c_{n}(y)|\Phi(n)-\Phi(N)|<(\mu+\nu)\left(e^{\nu}-e^{-\mu}\right)+\epsilon,
$$

for all sufficiently large $y, M, N$ and any pair $\mu, \nu$ chosen suitably large.

Next we have to consider

$$
\begin{aligned}
\sum_{n=M}^{\infty} c_{n}(y)|\Phi(n)-\Phi(M)| & =\sum_{n=M}^{\infty} \lambda_{n}(y)\{\Phi(n)-\Phi(M)\} \\
& \sim \frac{1}{\log y} \sum_{n=M}^{\infty} \frac{\log \log n-\log \log M}{(n+1)} e^{-(n+1) / y \quad(y \rightarrow \infty)} \\
& <\frac{1}{\log y} \int_{M}^{\infty} \frac{\log \log u-\log \log M}{u} e^{-u / y} d y=S_{3} \text { (say). }
\end{aligned}
$$

The integral in (39) is the same as that in (29). And the treatment of this integral in the steps following (29) and ending with (33) shows that

$$
\begin{aligned}
S_{3}<\log \frac{\log y}{\log M} \cdot\left(1-\frac{\log M}{\log y}\right)+ \\
\quad+\frac{\log 2+\log (\log y / \log M)}{\log y} \int_{1}^{\infty} \frac{e^{-v}}{v} d v+o(1) \quad(y \rightarrow \infty) \\
=\mu\left(1-e^{-\mu}\right)+o(1)
\end{aligned}
$$

under the condition (36). Using the above upper estimate for $S_{3}$ in (39) we get at once 


$$
\sum_{n=M}^{\infty} c_{n}(y)|\Phi(n)-\Phi(M)|<\mu\left(1-e^{-\mu}\right)+\epsilon
$$

for $c_{n}(y), \Phi(u)$ and $y, M, N, \mu, \nu$ as in (38).

(38) and (40) together show that hypothesis (12) of Proposition 2 is satisfied as a special case in Theorem III $(L)$. And so the deduction of Theorem III $(L)$ from Proposition 2 is complete.

4. Concluding remarks. Ishiguro's Tauberian theorem ([4], Theorem 2), already referred to, is an analogue of the original Tauberian theorem that, for any sequence $\left\{s_{n}\right\}$ summability $(A)$ and the condition $\lim n\left(s_{n}-s_{n-1}\right)=0$ together imply convergence. Theorems $\mathrm{I}(A), \mathrm{I}(L)$ and Theorems $\operatorname{III}(A)$, III $(L)$ of the present paper are analogues of two successive developments of the original Tauberian theorem, the first of which assumes that $\left\{s_{n}\right\}$ is summable $(A)$ and $\lim \inf n\left(s_{n}-s_{n-1}\right) \geqq 0$ but finite, and the second assumes that $\left\{s_{n}\right\}$ is bounded $(A)$ and $\lim \inf n\left(s_{n}-s_{n-1}\right)=0$. The result last referred to arises out of Vijayaraghavan's well-known extension of the original Tauberian theorem, to the effect that a sequence $\left\{s_{n}\right\}$ is bounded when it is bounded $(A)$ and $\lim \inf n\left(s_{n}-s_{n-1}\right) \geqq 0$. And there is a complement to Vijayaraghavan's extension, due to Karamata [6] and mentioned earlier, in which the condition that $\left\{s_{n}\right\}$ is bounded $(A)$ is replaced by the condition that $\left\{s_{n}\right\}$ is bounded $(A)$ on the right. Theorems $\mathrm{II}(A)$ and $\mathrm{II}(L)$, in their alternative (i) are analogous to Vijayaraghavan's theorem, while, in their alternative (ii), they are analogous to Karamata's theorem.

\section{REFERENCES}

[1] BoRwein, D., Theorems on some methods of summabilty, Quart. Journ. Math. Oxford Ser. (2), 9(1958), 310-316.

[2] HARDY, G. H., Divergent Series, (Oxford, 1949).

[3] ISHIGURO, K., A converse theorem on the summability methods, Proc. Japan Acad., $39(1963), 38-41$.

[4] ISHIGURO, K., Tauberian theorems concerning the summability methods of logarithmic type, Proc. Japan Acad., 39(1963), 156-159.

[5] JAKIMOVski, A. (AMNON AMIR), Some relations between the methods of summability of Abel, Borel, Cesàro, Hölder and Hausdorff, Journ. d'Analyse Math., 3(1953-54), 346-381.

[6] Karamata, J., Complément à un théorème de M. Hadwiger, Comment. Math. Helv., 25(1951), 64-70.

[ 7 ] Rajagopal, C.T., Tauberian oscillation theorems, Compositio Math., 11(1953), 71-82.

[8] Rajagopal, C.T. and Vijayaraghavan, T., One sided Tauberian theorems for Borel, Abel and Riemann-second-order transforms, Rend. Circ. Mat. Palermo (2), 4(1955), 307-322.

RAMANUJAN INSTITUTE OF MATHEMATICS

UNIVERSITY OF MADRAS, INDIA 